\title{
Flat-spectrum radio sources as likely counterparts of unidentified INTEGRAL sources (Research Note)
}

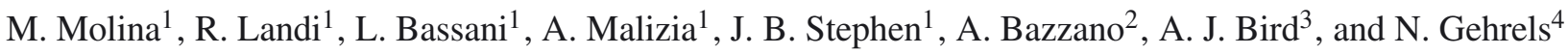 \\ ${ }^{1}$ IASF/INAF, via Gobetti 101, 40129 Bologna, Italy \\ e-mail: molina@iasfbo.inaf.it \\ 2 IAPS/INAF, via del Fosso del Cavaliere 100, 00133 Rome, Italy \\ 3 School of Physics and Astronomy, University of Southampton, SO17 1BJ, Southampton, UK \\ ${ }^{4}$ NASA Goddard Space Flight Center, Greenbelt, MD 20771
}

Received 4 May 2012 / Accepted 5 October 2012

\begin{abstract}
Many sources in the fourth INTEGRAL/IBIS catalogue are still unidentified since they lack an optical counterpart. An important tool that can help in identifying and classifying these sources is the cross-correlation with radio catalogues, which are very sensitive and positionally accurate. Moreover, the radio properties of a source, such as the spectrum or morphology, could provide further insight into its nature. In particular, flat-spectrum radio sources at high Galactic latitudes are likely to be AGN, possibly associated to a blazar or to the compact core of a radio galaxy. Here we present a small sample of 6 sources extracted from the fourth INTEGRAL/IBIS catalogue that are still unidentified or unclassified, but which are very likely associated with a bright, flat-spectrum radio object. To confirm the association and to study the source X-ray spectral parameters, we performed X-ray follow-up observations with Swift/XRT of all objects. We report in this note the overall results obtained from this search and discuss the nature of each individual INTEGRAL source. We find that 5 of the 6 radio associations are also detected in X-rays; furthermore, in 3 cases they are the only counterpart found. More specifically, IGR J06073-0024 is a flat-spectrum radio quasar at $z=1.08$, IGR J14488-4008 is a newly discovered radio galaxy, while IGR J18129-0649 is an AGN of a still unknown type. The nature of two sources (IGR J07225-3810 and IGR J19386-4653) is less well defined, since in both cases we find another X-ray source in the INTEGRAL error circle; nevertheless, the flat-spectrum radio source, likely to be a radio loud AGN, remains a viable and, in fact, a more convincing association in both cases. Only for the last object (IGR J11544-7618) could we not find any convincing counterpart since the radio association is not an X-ray emitter, while the only X-ray source seen in the field is a G star and therefore unlikely to produce the persistent emission seen by INTEGRAL.
\end{abstract}

Key words. gamma rays: galaxies - X-rays: galaxies - galaxies: active - quasars: general

\section{Introduction}

One of the key objectives of the INTEGRAL mission (Winkler et al. 2003) is to survey the sky at high energies (>20 keV), where non-thermal processes take place, the effects of absorption are drastically reduced, and extreme astrophysical phenomena are observed. This survey is performed by taking advantage of the unique imaging capabilities of the IBIS instrument (Ubertini et al. 2003), which allows the detection of sources at the mCrab flux level, with an angular resolution of $12^{\prime}$ and a point source location accuracy of typically $1-3^{\prime}$ within a large $(29 \times 29$ degrees) field of view.

Up to now, several surveys have been compiled from data collected by IBIS, the largest one being the fourth catalogue by Bird et al. (2010), which lists 723 hard X-ray sources of both Galactic and extragalactic nature. However, many objects in this survey are still unidentified, i.e. they have no obvious counterpart in other wavebands and so cannot be properly classified. Their classification remains one of the main objectives of the survey work, but it is made difficult by the large IBIS positional uncertainty. For this reason, accurate arcsecond localisation is pivotal to pinpoint their likely optical counterpart and assess their nature by means of spectroscopic follow-up observations (e.g.
Masetti et al. 2012, and references therein). Furthermore, many of these unidentified INTEGRAL sources are newly discovered and therefore lack multiwaveband data, which could provide important clues to their overall properties, hence nature.

An important tool that can help in identifying these sources is the cross-correlation with catalogues in other wavebands, for example in the radio. There are many valid reasons to use radio catalogues for counterpart searches: some high-energy emitting objects are also radio sources, such as active galactic nuclei (AGN), pulsars/pulsar wind nebulae, and X-ray binaries; radio surveys are very sensitive and positionally very accurate; the radio band does not suffer from the absorption which maybe a limitation in other wavebands, including the soft X-ray one. Moreover, the radio properties of a source, such as the spectrum or morphology, could provide further insight into their nature. In particular, flat-spectrum radio sources at high galactic latitudes are likely to be AGN, possibly associated to a blazar or to the compact core of a radio galaxy.

In this work, we present a sample of six unidentified INTEGRAL/IBIS sources, selected on the basis of their association with a radio source showing a flat spectrum. To confirm the radio/INTEGRAL association and provide broad-band spectral information on these objects, we have also performed X-ray 
Table 1. The sample: INTEGRAL/IBIS data and radio associations.

\begin{tabular}{lccccccccc}
\hline \hline Name & RA & Dec & $\begin{array}{c}\text { Pos. err. } \\
\text { (arcmin) }\end{array}$ & Burst. & $F_{20-40^{\dagger}}$ & $N_{\mathrm{H}}$ & \multicolumn{2}{c}{ Radio coordinates $^{\dagger}$} & $\alpha_{\text {radio }}$ \\
& & & & & $10^{22} \mathrm{~cm}^{-2}$ & RA (J2000) & Dec (J2000) & \\
\hline IGR J06073-0024 & 91.830 & -0.415 & 4.8 & $\mathrm{YY}$ & $1.21(5.98)^{*}$ & 0.23 & 060657.44 & -002457.5 & $-0.15^{C}$ \\
IGR J07225-3810 & 110.621 & -38.168 & 5.3 & $\mathrm{Y}$ & $0.98(1.74)^{*}$ & 0.21 & 072222.00 & -381455.0 & $-0.26^{C}$ \\
IGR J11544-7618 & 178.592 & -76.309 & 5.0 & & 0.68 & 0.08 & 115547.00 & -761908.0 & $+0.82^{C}$ \\
IGR J14488-4008 & 222.209 & -40.142 & 4.4 & & 0.38 & 0.07 & 144851.01 & -400845.7 & $-0.03^{C}$ \\
IGR J18129-0649 & 273.224 & -6.829 & 4.1 & $\mathrm{Y}$ & $0.30(0.45)^{*}$ & 0.35 & 181250.95 & $-064823.8^{\ddagger}$ & $-0.49^{S}$ \\
IGR J19386-4653 & 294.653 & -46.886 & 4.5 & $\mathrm{YY}$ & $<0.30(5.30)^{*}$ & 0.05 & 193826.32 & -465725.6 & $+0.26^{C}$ \\
\hline
\end{tabular}

Notes. ${ }^{(\dagger)}$ Fluxes are expressed in units of $10^{-11} \mathrm{erg} \mathrm{cm}^{-2} \mathrm{~s}^{-1}$. $^{(*)}$ The flux reported in parenthesis refers to the peak flux in $20-40 \mathrm{keV}$ band for sources detected through the bursticity analysis (see text for details). (\$) Radio position from the NVSS catalogue (see text). Energy spectral index from $\mathrm{C}=$ CRATES (estimated in the range 0.843/1.4 and 4.85 GHz); $\mathrm{S}=$ SPECFIND v.2.0.

follow-up observations with the X-ray telescope (XRT; Burrows et al. 2005) on board the Swift satellite (Gehrels et al. 2004). The radio and $\mathrm{X}$-rays properties are then combined with information gathered from the literature to identify the likely counterpart(s) and discuss the nature of each INTEGRAL source.

\section{The sample}

The sample selection was made on the basis of the crosscorrelation between the fourth INTEGRAL/IBIS catalogue (Bird et al. 2010) and several public radio catalogues, using the technique described in Stephen et al. $(2005,2006,2010)$ and fully tested in these papers. The radio catalogues employed in the cross-correlation analysis are from the following surveys: the Combined Radio All-sky Targeted Eight GHz Survey (CRATES; Healey et al. 2007), the Australia Telescope $20 \mathrm{GHz}$ Survey (AT20G; Murphy et al. 2010), the Sydney University Molonglo Sky Survey (SUMSS; Bock et al. 1999), and the VLA Lowfrequency Sky Survey (VLSS; Cohen et al. 2007). The crosscorrelation procedure returns a list of sources that at least have a counterpart in one of those catalogues. Among these sources, we selected six INTEGRAL objects that have not yet been identified in other wavebands and have an association with a flatspectrum radio source ${ }^{1}$. In particular, we find that five out of these six objects are listed in the CRATES catalogue, which is an all-sky survey of flat-spectrum radio sources. The remaining object (IGR J18129-0649, see Table 1) does not have a CRATES counterpart, but is reported to have a flat radio spectrum in the SPECFIND catalogue v.2.0 (Vollmer 2009). It is also the only object located within ten degrees of the Galactic plane, hence the only one for which an extragalactic nature is less secure. However, its Galactic latitude is above $|5|$ degrees, it is reported as a symmetric double in the Texas Survey of Radio sources (Douglas et al. 1996), and is variable in hard $\mathrm{X}$-rays (see below), all properties that hint toward an AGN close to the Galactic plane. For this reason we have retained IGR J18129-0649 within the set of sources analysed in this work. Finally, we note that two sources appear in more than one catalogue: IGR J14488-4008 in CRATES and SUMSS and IGR J18129-0649 in AT20G and VLSS.

The sample is presented in Table 1, where we list in the first six columns the INTEGRAL name, coordinates, $90 \%$ positional error radius, bursticity flag if any, $20-40 \mathrm{keV}$ flux, and Galactic column density in the direction of each source (Kalberla et al. 2005). Hard X-ray information is all taken from

\footnotetext{
1 A flat spectrum radio source is one with energy index $\alpha \geq-0.5$, assuming $\mathrm{S}_{v} \propto v^{\alpha}$.
}

Bird et al. (2010). The bursticity of a source is defined as the ratio of the maximum significance on any timescale, compared to the significance defined for the whole data set analysed (see Bird et al. 2010, for details). A bursticity of one defines a persistent source, while a bursticity greater than one (flagged $\mathrm{Y}$ in the Bird et al. survey) implies that the significance of a source is increased by the omission of some observations from the analysis, presumably when the source was in quiescence. A bursticity greater than four (flagged YY) instead indicates a strongly variable source. We note that all but two objects are classified as variable in the fourth INTEGRAL/IBIS catalogue; the two exceptions are IGR J11544-7618 and IGR J14488-4008, which are reported as persistent sources. In the last three columns of Table 1, we list the coordinates of the flat-spectrum radio association found and its energy spectral index. The radio positions are taken from CRATES, except for IGR J18129-0649, for which we use the NVSS coordinates (Condon et al. 1998). The radio spectral indices are all from CRATES (estimated between $0.843 / 1.4$ and $4.85 \mathrm{GHz}$ ), except for IGR J18129-0649, for which the SPECFIND catalogue v.2.0 has been used instead (Vollmer 2009).

\section{Swift observations and data reduction}

To assess the likelihood of the INTEGRAL/radio association and to obtain information over a wide range of frequencies, we requested and obtained follow-up observations of all six objects with the X-ray telescope (XRT; Burrows et al. 2005) on board the Swift satellite (Gehrels et al. 2004). We note that no X-ray data were available for these INTEGRAL sources prior to the XRT pointings. For each source, one or more measurements were performed as reported in Table 2; multiple observations were summed together to enhance the signal-to-noise ratio. XRT data reduction was done using the XRTDAS standard data pipeline package (XRTPIPELINE v.0.12.6 included in the HEASOFT package v.6.12) ${ }^{2}$ in order to produce screened event files. All data were extracted only in the photon counting (PC) mode (Hill et al. 2004), by adopting the standard grade filtering (0-12 for PC) according to the XRT nomenclature.

The XRT images extracted in the $0.3-10 \mathrm{keV}$ band were searched for significant excesses (above $2.5 \sigma$ level) falling within the IBIS positional uncertainty or close to it. One out of ten counterparts is in fact expected to fall just outside the $90 \%$ IBIS error circle. Further details on the image analysis

2 The XRT Data Analysis Software (XRTSDAS) was developed by the ASI Science Data Center (ASDC), Italy in collaboration with HEASARC NASA/GSFC and is available at

http://heasarc.gsfc.nasa.gov/docs/swiftanalysis/ 


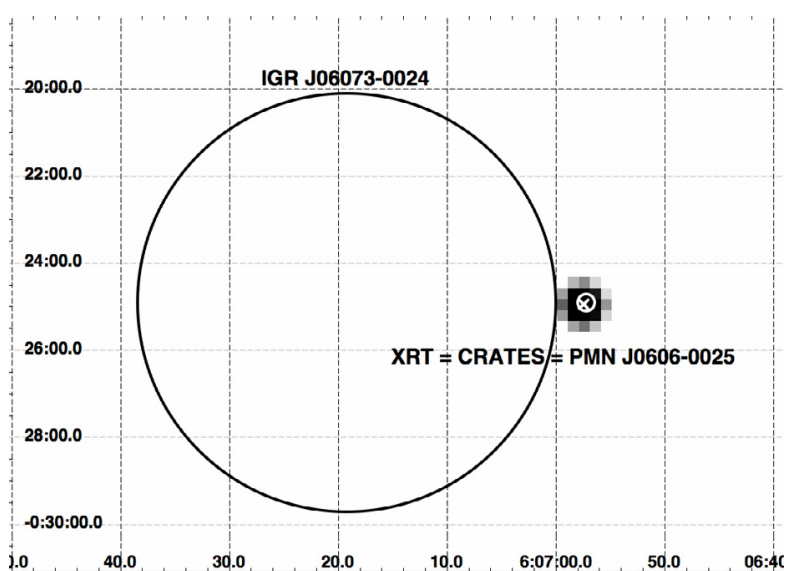

Fig. 1. NVSS $1.4 \mathrm{GHz}$ image of the region surrounding IGR J06073-0024. The large circle refers to the IBIS positional uncertainty, and the smaller one to the XRT position, which is coincident with the CRATES/PMN source (indicated with an X-shaped symbol).

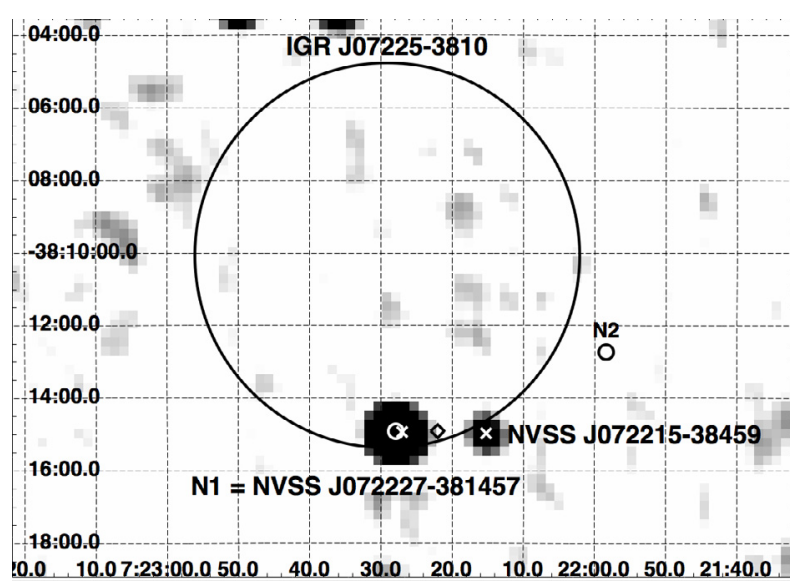

Fig. 2. NVSS $1.4 \mathrm{GHz}$ image of the region surrounding IGR J07225-3810. The large circle refers to the IBIS positional uncertainty, and the smaller ones to the position of the two XRT detections. The $\mathrm{X}$-shaped symbol corresponds to the position of the radio counterpart. Also shown with a small diamond is the position of the CRATES/PMN source discussed in the text, which falls in between the two NVSS detections.

can be found in Landi et al. (2010), while the present results are reported in Table 2, where we list, besides the INTEGRAL source name and number of X-ray observations performed, the total exposure available, the position and relative uncertainty of each X-ray detection and the number of sigma found. Sources detected above $3 \mathrm{keV}$ are highlighted, since these are the most likely to be true counterparts of the IBIS sources. Figures 1 to 6 show the collection of NVSS/SUMSS image cut-outs for all sources, with the IBIS error circle and the position of the XRT detection(s) superimposed. In all but one case, that of IGR J11544-7618, the flat spectrum radio association reported in Table 1 has been detected in X-rays, suggesting that it could be a viable counterpart.

We also studied the X-ray spectral properties of each source in order to gain further information. Events for spectral analysis were extracted within a circular region of radius $20^{\prime \prime}$, centered on the source position, which encloses about $90 \%$ of the PSF at $1.5 \mathrm{keV}$ (see Moretti et al. 2004). The background was taken from various source-free regions close to the X-ray source of

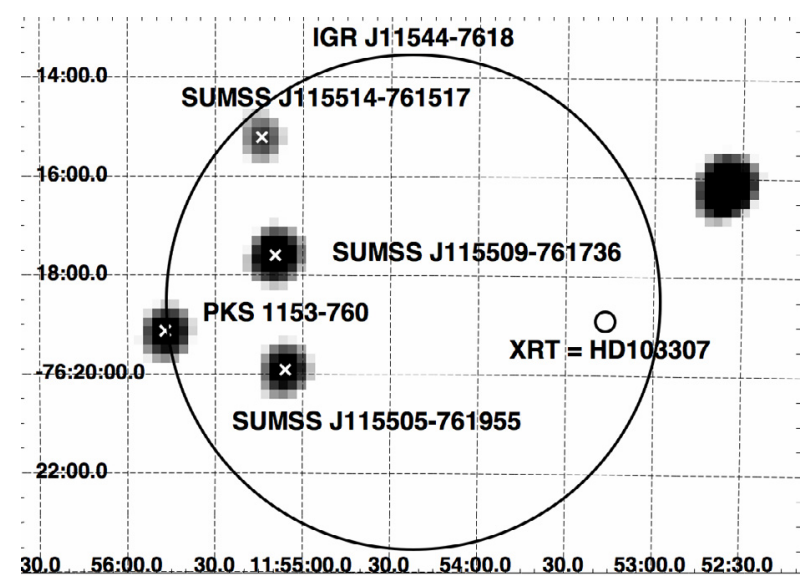

Fig. 3. SUMSS $0.84 \mathrm{GHz}$ image of the region surrounding IGR J11544-7618. The large circle refers to the IBIS positional uncertainty, and the smaller one to the XRT position of the star HD103307. Also highlighted are the position of the CRATES source (PKS 1153-780) and of 3 SUMSS detections all marked by X-shaped symbols.

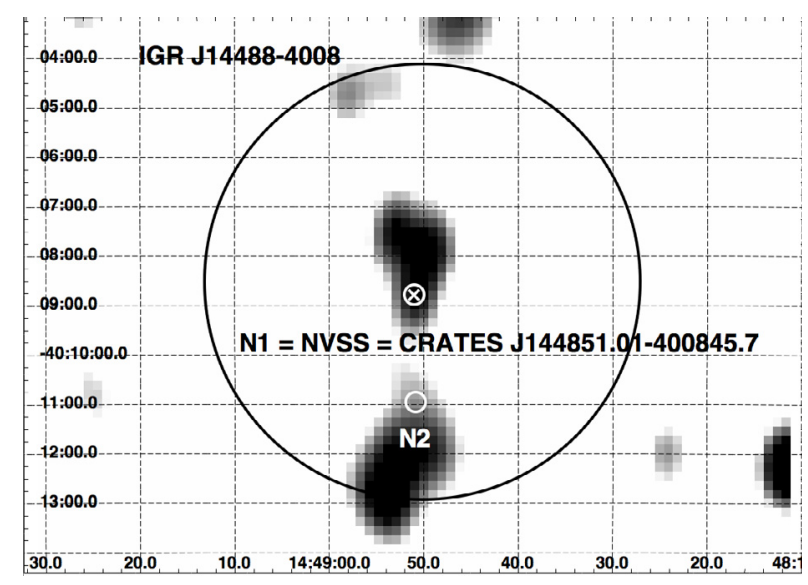

Fig. 4. SUMSS $0.84 \mathrm{GHz}$ image of the region surrounding IGR J14488-4008. The large circle refers to the IBIS positional uncertainty, and the smaller ones to the the position of the two XRT detections. The X-ray source N1 coincides with one of the radio excesses associated to CRATES source (indicated with an X-shaped symbol) and likely representing the compact core of a radio galaxy.

interest, using circular regions with different radii in order to ensure an evenly sampled background. Spectra were extracted from the corresponding event files using the XSELECT software and binned using the FTOOL grppha in an appropriate way, so that the $\chi^{2}$ statistic could be applied. We used version v.011 of the response matrices and created individual ancillary response files arf using xrtmkarf v. 0.5.9.

Analysis of these spectra was performed in the $0.3-6 \mathrm{keV}$ energy range, using XSPEC version 12.5.1 (Arnaud 1996). The data were fitted using a simple power law absorbed by the Galactic column density measured in the direction of each source and reported in Table 1. Errors on photon index are quoted at $90 \%$ confidence level for one parameter of interest $\left(\Delta \chi^{2}=2.71\right)$. The results of the spectral analysis are reported in the last two columns of Table 2, where we list the photon index and the estimated $2-10 \mathrm{keV}$ flux. 
Table 2. The sample: Swift/XRT data.

\begin{tabular}{|c|c|c|c|c|c|c|c|c|c|}
\hline Name & N. Obs & $\begin{array}{c}\text { Exposure } \\
\text { (s) }\end{array}$ & RA & Dec & $\begin{array}{l}\text { Pos. err. } \\
(\operatorname{arcsec})\end{array}$ & $\sigma$ & Radio Ass. ${ }^{\star}$ & $\Gamma$ & $\begin{array}{c}F_{2-10 \mathrm{keV}} \\
\mathrm{erg} \mathrm{cm}^{-2} \mathrm{~s}^{-1}\end{array}$ \\
\hline IGR J06073-0024 & 4 & 3961 & 060657.20 & -002454.70 & 6.0 & 3.0 & Y & $1.8 \mathrm{f}$ & $1.10 \times 10^{-13}$ \\
\hline \multirow{2}{*}{ IGR J07225-3810 } & 3 & 4488 & 072227.90 & $-381455.39(\mathrm{~N} 1)$ & 4.7 & $7.0^{\dagger}$ & $\mathrm{Y}$ & $1.52_{-0.46}^{+0.45}$ & $5.76 \times 10^{-13}$ \\
\hline & & & 072158.30 & $-381244.40(\mathrm{~N} 2)$ & 6.0 & $4.1^{\dagger}$ & $\mathrm{N}$ & $1.8 \mathrm{f}$ & $1.1 \times 10^{-13}$ \\
\hline IGR J11544-7618 & 2 & 4186 & 115316.46 & -761854.98 & 5.5 & 4.6 & $\mathrm{~N}$ & $1.8 \mathrm{f}$ & $6.18 \times 10^{-14}$ \\
\hline \multirow[t]{2}{*}{ IGR J14488-4008 } & 3 & 8784 & 144850.97 & $-400847.01(\mathrm{~N} 1)$ & 3.8 & $14.1^{\dagger}$ & $\mathrm{Y}$ & $2.20_{-0.68}^{+0.68}$ & $2.53 \times 10^{-12}$ \\
\hline & & & 144850.82 & $-401056.70(\mathrm{~N} 2)$ & 4.6 & 5.8 & $\mathrm{~N}$ & $1.8 \mathrm{f}$ & $1.42 \times 10^{-13}$ \\
\hline IGR J18129-0649 & 1 & 1314 & 181250.68 & -064825.11 & 4.6 & $7.9^{\dagger}$ & Y & $1.44_{-0.43}^{+0.41}$ & $2.74 \times 10^{-12}$ \\
\hline \multirow[t]{2}{*}{ IGR J19386-4653 } & 1 & 5114 & 193859.80 & $-465124.20(\mathrm{~N} 1)$ & 6 & 3.0 & $\mathrm{~N}$ & $1.8 \mathrm{f}$ & $8.00 \times 10^{-14}$ \\
\hline & & & 193826.20 & $-465720.10(\mathrm{~N} 2)$ & 6 & 2.7 & Y & $1.8 \mathrm{f}$ & $6.00 \times 10^{-14}$ \\
\hline
\end{tabular}

Notes. ${ }^{(\dagger)}$ Source detected also above $3 \mathrm{keV} .{ }^{(\star)}$ Confirmed association between X-ray detection and radio counterpart listed in Table 1.

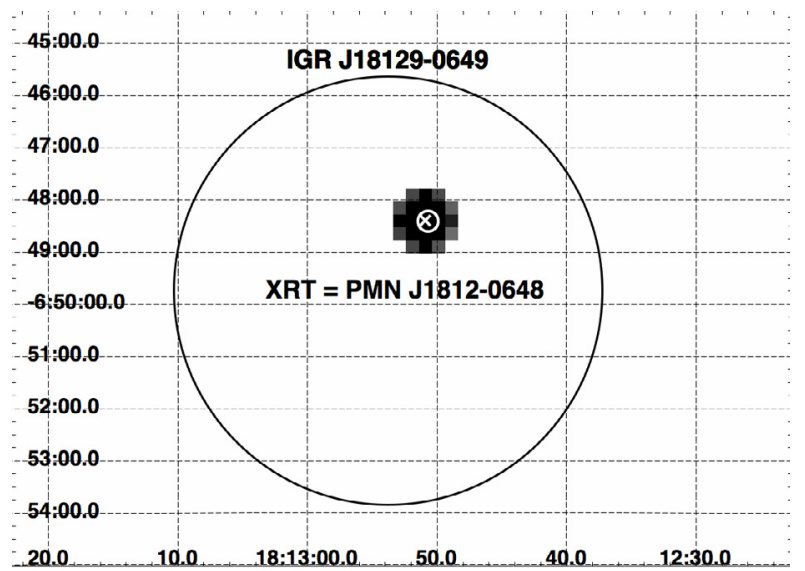

Fig. 5. NVSS $1.4 \mathrm{GHz}$ image of the region surrounding IGR J18129-0649. The large circle refers to the IBIS positional uncertainty, and the smaller one to the XRT position, which is coincident with a radio source PMN J1812-0648 (indicated with an X-shaped symbol) detected in various catalogues.

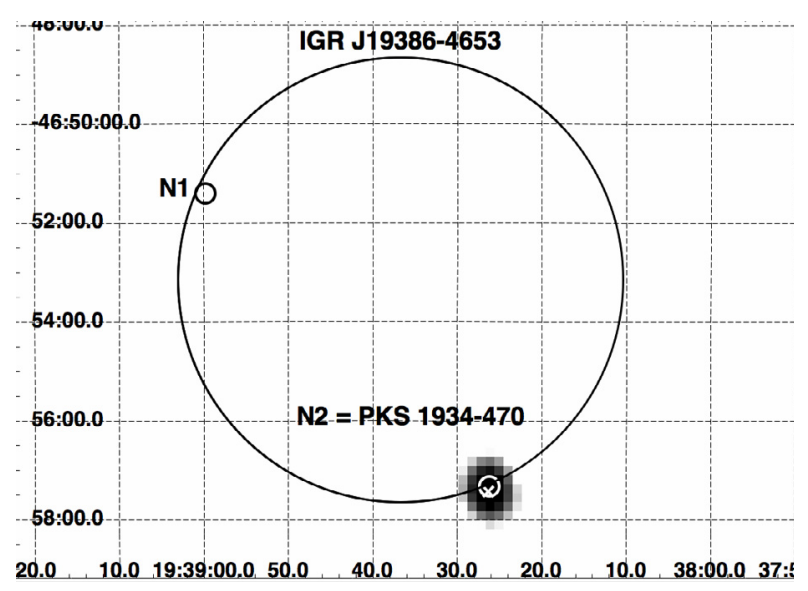

Fig. 6. SUMSS $0.8 \mathrm{GHz}$ image of the region surrounding IGR J19386-4653. The large circle refers to the IBIS positional uncertainty, and the smaller ones to the the position of the two XRT detections. The X-ray source N1 is not detected in radio, while source $\mathrm{N} 2$ is coincident with the CRATES/PKS source (indicated with an $\mathrm{X}$-shaped symbol).

\section{Source details}

In the following, we examine each individual INTEGRAL source in detail to assess the likelihood of the $\mathrm{X} /$ radio association and understand the true nature of each object. To this end, we use all the available multiwavelength information in the literature or in publicly available databases; we also analyse the source radio loudness, defined as $\log \left(R_{L}\right)=\log \left(L_{5 \mathrm{GHz}} / L_{B}\right)^{3}$, where $L_{5} \mathrm{GHz}$ is the luminosity at $5 \mathrm{GHz}$ and $L_{B}$ the luminosity in the $B$ band. We also use the $S_{I}$ statistics developed by Edelson \& Malkan (2012) and based on WISE (Wide-Field Infrared Survey Explorer; Wright et al. 2010) and 2MASS (Two Micron All-Sky Survey; Skrutskie et al. 2006) data to reliably identify IGR J18129-0649 as an AGN: sources for which $S_{I}$ is below 1.73 have a $95 \%$ likelihood of being an AGN of type 1 (Seyfert1, quasar or blazar). WISE colours have also been used to probe the blazar likelihood of some objects (Massaro et al. 2012). In fact, in the [3.4]-[4.6]-[12] micron colour-colour diagram, Blazars cover a distinct region, hereafter the WISE blazar strip (WBS; see also Fig. 1 in Massaro et al. 2012): if a source falls within or close to the WBS, it can potentially be an AGN of the blazar type.

\subsection{IGR J06073-0024}

Just outside the INTEGRAL 90\% error circle (see Fig. 1 and Table 2), we find an X-ray source that is consistent with the counterpart found in the CRATES catalogue and reported in NED as PMN J0606-0025. It is detected at several radio frequencies from 1.4 to $8.4 \mathrm{GHz}$ and shows a compact radio morphology (see Fig. 1). It has a NVSS $1.4 \mathrm{GHz}$ flux of $133.9 \pm 4 \mathrm{mJy}$, it is detected at $5 \mathrm{GHz}$ in the Parkes-MIT-NRAO (PMN; Griffith et al. 1995) survey with a flux of $116 \pm 12 \mathrm{mJy}$ and in the MITGreen Bank survey (Bennett et al. 1986) with a flux of $96 \mathrm{mJy}$, therefore some degree of flux variability at this frequency cannot be ruled out. In the CRATES catalogue, the source is reported to have an $8.4 \mathrm{GHz}$ flux of $\sim 98 \mathrm{mJy}$, which combined with the $1.4 \mathrm{GHz}$ flux, provides the flat spectral index reported in Table 1. The $4.85 \mathrm{GHz}$ flux can be used together with the $B$ magnitude to estimate the source radio loudness. Indeed, coincident with the radio and X-ray positions, we find an optical counterpart in the USNO B-1 catalogue (Monet et al. 2003), with magnitude $B=20.2$ (or $0.02 \mathrm{mJy}$ in flux); here and in the following we use the standard photometric system conversion from magnitude to flux (Zombeck 1990). The radio loudness therefore lies in the range 3.7-3.8.

The CRATES source has recently been optically classified and found to be a broad emission line AGN (FWHM = $5600 \mathrm{~km} \mathrm{~s}^{-1}$ ) at $z=1.028$ (Masetti et al. 2012). This initially

3 Radio loud AGN have $\log \left(R_{L}\right)$ values greater than 1 (Kellermann et al. 1989). 
tentative identification is now supported by the X-ray detection. The statistical quality of the X-ray spectrum is such that we can only estimate a $2-10 \mathrm{keV}$ flux of $1.1 \times 10^{-13} \mathrm{erg} \mathrm{cm}^{2} \mathrm{~s}^{-1}$. At the reported redshift and assuming $H_{0}=71 \mathrm{~km} \mathrm{~s}^{-1} \mathrm{Mpc}^{-1}$, $\Omega_{\Lambda}=0.73$ and $\Omega_{\mathrm{M}}=0.27$, the source X-ray luminosities are $3.8 \times 10^{43} \mathrm{erg} \mathrm{s}^{-1}$ and $4.1 \times 10^{45} \mathrm{erg} \mathrm{s}^{-1}$ in the $2-10 \mathrm{keV}$ and $20-40 \mathrm{keV}$ band, respectively; this suggests that the IGR J06073-0024 X-ray spectrum rises towards higher frequencies, as expected in flat spectrum radio quasars. Masetti and coworkers also estimate the mass of the black hole at the centre of this object to be $5 \times 10^{8} M_{\odot}$, i.e. quite a massive black hole, similar to those found in other high-redshift AGN discovered in hard X-ray surveys (Ghisellini et al. 2010; De Rosa et al. 2012).

The source is also reported in the WISE survey (Wright et al. 2010) with the following magnitudes $W_{1}=14.99(3.4 \mu \mathrm{m})$, $W_{2}=14.03(4.6 \mu \mathrm{m}), W_{3}=11.51(12 \mu \mathrm{m})$ and $W_{4}=8.83$ $(22 \mu \mathrm{m})$. Its WISE colours are $W_{2}-W_{3}=2.52$ and $W_{1}-W_{2}=$ 0.96 , therefore placing it in the WBS. We therefore conclude that IGR J06073-0024 is a newly discovered flat spectrum radio quasar at high $z$, hence a new hard X-ray selected blazar.

\subsection{IGR J07225-3810}

In this case, we find two potential X-ray counterparts (see Table 2): one (N1 in Fig. 2 and Table 2) lies inside the IBIS error circle, while the other (N2) is outside its border. Source N1 is also the brightest of the two and the only one detected above $3 \mathrm{keV}$ with some confidence (above 2.5 sigma); this, together with its location inside the INTEGRAL positional uncertainty, makes it a more convincing counterpart to the INTEGRAL source. Besides, we could not find any obvious association for source N2, so this object cannot be discussed further.

Source N1 coincides instead with the CRATES association proposed in Table 1 and reported in NED as PMN J0722-3814. As is evident in Fig. 2, this CRATES source is slightly offset from the bright radio source (NVSS J072227-381457) falling inside the INTEGRAL error circle. This offset may be due to the inability of the PMN survey (used as a reference for the CRATES position) to resolve the emission in two nearby objects as done by the NVSS (see Fig. 2). In the following, we assume that the bright NVSS source and the CRATES/PMN object are the same: in this case the $1.4 \mathrm{GHz}$ (NVSS) and $0.843 \mathrm{GHz}$ (SUMSS) fluxes are 135 and $203 \mathrm{mJy}$ respectively, while the flux at $4.85 \mathrm{GHz}$ (PMN) is $\leq 110 \mathrm{mJy}$ (PMN) (due to possible contamination from the nearby and dim NVSS detection). The 1 to $5 \mathrm{GHz}$ spectral index is around $-0.2 /-03$, i.e. compatible with that reported in Table 1. Consistent with the NVSS/XRT positions, we find an optical USNO B-1 source with optical magnitude $B=19.1$ (0.1 mJy in flux), which provides $\log \left(R_{L}\right) \leq 3$.

The XRT spectrum for source N1 has enough statistics to allow determination of the photon index, which, despite the large errors, is rather flat, at $1.52_{-0.46}^{+0.45}$, the X-ray $2-10 \mathrm{keV}$ flux is $5.76 \times 10^{-13} \mathrm{erg} \mathrm{cm}^{2} \mathrm{~s}^{-1}$. The source is also detected by WISE with the following magnitudes: $W_{1}=14.67, W_{2}=13.61$, $W_{3}=10.22$ and $W_{4}=8.33$. Its WISE colours, $W_{2}-W_{3}=3.39$, and $W_{1}-W_{2}=1.06$, locate this source on the WISE blazar strip in a region compatible with the colours of flat spectrum radio quasars. We therefore conclude that source N1 is an AGN possibly of the blazar type given its overall properties (flat X-ray spectrum, WISE colours, and radio characteristics); as such, it is a convincing counterpart of the variable INTEGRAL source. The nature of object N2 is unclear at this stage, but its location, as well as X-ray weakness, makes it a less convincing association.

\subsection{IGR J11544-7618}

The region surrounding IGR J11544-7618 is quite complex at radio frequencies. Apart from the CRATES source reported in Table 1 and listed in NED as PKS 1153-783, there are three other objects from the SUMSS survey falling within the INTEGRAL error circle (see Fig. 3). None of these objects is detected in X-rays by XRT, placing a limit on their X-ray emission at around $3 \times 10^{-14} \mathrm{erg} \mathrm{cm}^{2} \mathrm{~s}^{-1}$.

However, inside the IBIS error circle there is an X-ray source that is not coincident with any radio emission (see Fig. 3) and previously reported as a Rosat faint source (1RXS J115313.6$\left.761935^{4}\right)$; the X-ray spectrum from this source is soft, since it is not detected above $3 \mathrm{keV}$. The XRT coordinates coincide with a bright star, HD103307 of spectral type G0/3IV/V. Solarlike stars like HD103307 emit X-rays via coronal emission, but are unlikely to be detected at energies higher than a few $\mathrm{keV}$. However, they sometimes show activity in the form of energetic flares, and these stellar flares radiate at all wavelengths from radio to gamma-rays, last typically from minutes to hours/days, and have been occasionally detected by IBIS as variable objects. However, IGR J11544-7618 is a persistent source in the 20-40 keV band, so we can exclude that the emission detected by INTEGRAL is due to flares coming from this star.

At the same time we cannot invoke X-ray variability to explain the lack of X-ray emission from the CRATES or any other radio source in the region. Unfortunately, the counterpart of this INTEGRAL object remains unclear, and only further follow-up observations in various wavebands would be able to shed some light on its nature. Another possibility that cannot be ruled out at the moment is that IGR J11544-7618 is a spurious detection by IBIS.

\subsection{IGR J14488-4008}

As is evident from Fig. 4, the CRATES source listed in Table 1 has an extremely complex radio morphology, with multiple structures that resemble a radio galaxy with two bright lobes fully contained within the INTEGRAL error circle. Also in this case, there are two X-ray sources (N1 and N2 in Table 2 and in Fig. 4) falling within IBIS positional uncertainty; one of the two (N1) is brighter and harder since it is also detected above $3 \mathrm{keV}$. This source is associated to one of the CRATES excesses (CRATES J1448-4008), as well as to one of the NVSS (NVSS $\mathrm{J} 144851-400846$ ) peaks reported in this region. This excess is located at the centre of the radio structure and is also coincident with the optical position of the main galaxy, PGC 589690 (Paturel et al. 2003). N1 could therefore be the compact core of a newly discovered radio galaxy.

Because of the complex structure, it is difficult to combine radio observations at various frequencies to estimate a spectral index. If we confine the search to radio emission within the $\mathrm{X}$-ray error circle of source $\mathrm{N} 1$, we find that it is detected at 1.4, $4.85,8.4$, and $8.6 \mathrm{GHz}$ with fluxes of $48,48,45.9$, and $46 \mathrm{mJy}$ respectively (NVSS; CRATES; ATPMN; McConnell et al. 2012). This provides a spectral index similar to the one reported in Table 1 . In the optical band, we find a counterpart in the USNOB1 catalogue having $B \sim 14.2$ (or $8.9 \mathrm{mJy}$ in flux); the radio loudness parameter is therefore 0.73 , making this object radio quiet.

\footnotetext{
4 The Rosat Faint Catalogue is available at http: //www . xray .mpe.mpg.de/rosat/survey/rass-fsc/
} 
The X-ray data are well-fitted using a double power-law model with indices tight together and with $\Gamma=2.20$, consistent with what reported by Malizia et al. (2011). In addition to the Galactic absorption, the data require an intrinsic column density $N_{\mathrm{H}}=\left(6.2_{-1.9}^{+2.1}\right) \times 10^{22} \mathrm{~cm}^{-2}$, suggesting that it might be a type 2 AGN.

Source N2 cannot be characterised with the same detail. In $\mathrm{X}$-rays, it is much dimmer and softer than object N1 and is also variable, since seen in only one of the three XRT pointings, which contrasts with the persistent nature of IGR J14488-4008. It has a counterpart in the USNO-B1/2MASS catalogues, but no detection in radio. It is difficult to assess its nature at this stage, but its X-ray characteristics (flux variability, X-ray weakness, as well as X-ray spectral softness) suggest that this source is unlikely to be the counterpart of the persistent INTEGRAL object, thus making N1 the most likely association to IGR J14488-4008.

\subsection{IGR J18129-0649}

The radio source listed in Table 1 lies well within the INTEGRAL error box. It coincides with the only detection made by XRT in this region (see Table 2 for details), which suggests that it is a valid counterpart of the INTEGRAL source. It is quite bright at radio frequencies, with a flux ranging from $2720 \mathrm{mJy}$ at $0.074 \mathrm{GHz}$ (Cohen et al. 2007) to $339 \mathrm{mJy}$ at $20 \mathrm{GHz}$ (Murphy et al. 2010). The overall spectrum is shown in Fig. 7: it has an energy index $\alpha \sim-0.5$ from 0.365 to $20 \mathrm{GHz}$ with some deviation at low frequencies. The source, named PMN J1812-0648 in NED, is compact in the NVSS map, thus suggesting that the classification as a symmetric double in the Texas survey is probably incorrect.

Besides being the brightest radio source among our sample, it is also the strongest in X-rays with a $2-10 \mathrm{keV}$ flux of $2.7 \times$ $10^{-12} \mathrm{erg} \mathrm{cm}^{2} \mathrm{~s}^{-1}$. The X-ray spectrum is well-fitted by a simple power-law model with a flat photon index of 1.4.

As anticipated, this is the only object in the sample that is relatively close to the Galactic plane, hence the only one for which an AGN nature is uncertain. For this reason we used the method employed by Edelson \& Malkan (2012) to recognise AGN using simply WISE/2MASS colours. The source is listed in the WISE catalogue with magnitudes $W_{1}=13.13, W_{2}=11.80$, $W_{3}=9.19$, and $W_{4}=6.77$, and the 2MASS magnitudes are instead $J=16.72, H=15.19$ and $K=14.77$. Using the Edelson $\&$ Malkan (2012) formalism, we estimate $S_{I}=0.9$, i.e. a value below the cut of 1.73 used to reliably identified type 1 AGN. Furthermore, we note that the WISE colours are $W_{2}-W_{3}=2.6$ and $W_{1}-W_{2}=1.34$, placing the source outside the WiSE blazar strip in a region compatible with the location of quasars.

The source has no counterpart in the USNO-B1 catalogue, with only an upper limit to the $B$ magnitude of 20.7 (or $0.02 \mathrm{mJy}$ in flux). Combining the $4.85 \mathrm{GHz}$ flux of $766 \mathrm{mJy}$ measured by the PMN survey with that in the $B$ band gives a radio loudness parameter $\log \left(R_{L}\right) \geq 4.5$; i.e., the source is certainly radio-loud. While the association of PMN J1812-0648 with the INTEGRAL source is secure and its AGN nature validated by observational evidence, its optical class is unclear, and only optical follow-up observations can assess its true nature.

\subsection{IGR J19386-4653}

Inside the $90 \%$ IBIS positional uncertainty, XRT detects two weak sources (N1 and N2 in Table 2 and in Fig. 6), none of which

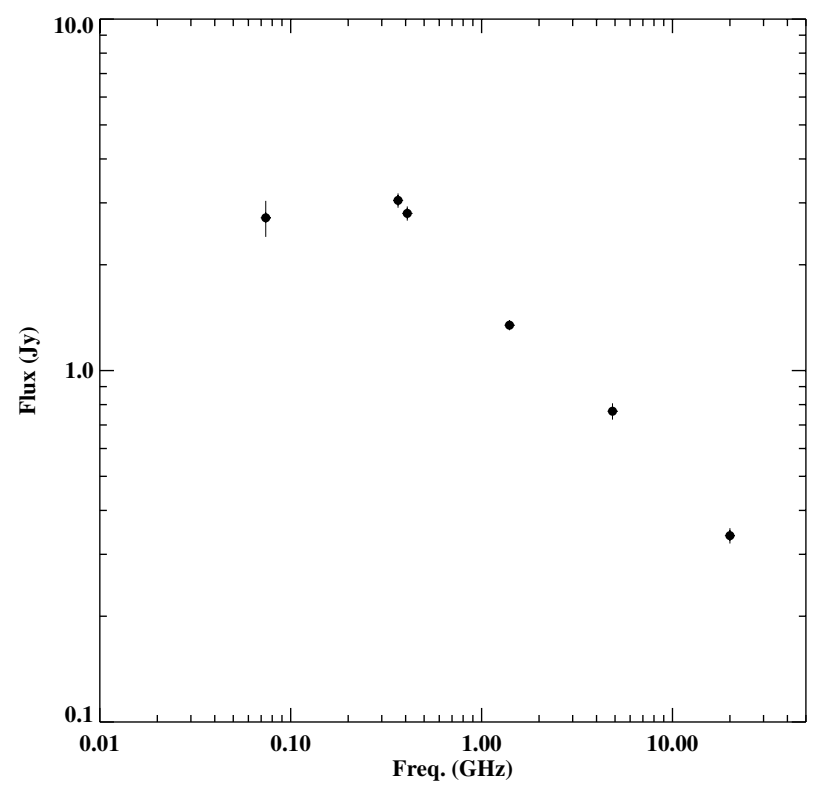

Fig. 7. Spectrum of the radio source PMN J1812-0648 associated to IGR J18129-0649. Data are from VLSS (Cohen et al. 2007), Texas (Douglas et al. 1996), Molonglo (Bock et al. 1999), NVSS (Condon 1992) PMN (Griffith et al. 1995), and AT20G (Murphy et al. 2010) surveys.

is visible above $3 \mathrm{keV}$. They are both quite dim in X-rays, with $2-10 \mathrm{keV}$ fluxes below $10^{-13} \mathrm{erg} \mathrm{cm}^{2} \mathrm{~s}^{-1}$. We remind the reader that IGR J19386-4653 is extremely variable in INTEGRAL, which could explain the weakness of both X-ray detections. The first source has two counterparts in the USNO-B1 and one in the 2MASS catalogues, but it is not detected in radio, nor it is listed in WISE.

The second one, N2, is instead associated with the CRATES object (also PKS 1934-470 in NED) listed in Table 1, thus suggesting that this is a viable counterpart to the INTEGRAL detection. Source N2 has been previously seen in X-rays since it is listed in the WGACAT catalogue (White et al. 2000), with a large positional uncertainty $\left(50^{\prime \prime}\right)$ that is, however, compatible with the XRT location. The WGACAT source is also reported in the Roma-Blazar catalogue (Massaro et al. 2009) and is classified by Landt et al. (2004) as a flat spectrum radio quasar at $z=0.8$; unfortunately, this source is located $35^{\prime \prime}$ from the XRT detection casting some doubts on their association. On the other hand, we could not find any optical counterpart within the XRT positional uncertainty, thus making the case even more puzzling.

At radio frequencies, source N2/PKS 1934-470 is well studied, with detections reported at various frequencies going from $138 \mathrm{mJy}$ at $0.843 \mathrm{GHz}$ (Bock et al. 1999) to $90 \mathrm{mJy}$ at $8.6 \mathrm{GHz}$ (McConnell et al. 2012); this provides a radio spectral index of -0.2 , still flat but substantially different from what is reported in Table 1. We note however that the value listed in the table is the one estimated in the 0.843 and $4.85 \mathrm{GHZ}$ band; when a wider frequency range is considered the index becomes -0.22 CRATES ( $\alpha_{2}$ in CRATES). The radio morphology is that of a compact source. At $5 \mathrm{GHz}$ the reported fluxes range from $104 \mathrm{mJy}$ (McConnell et al. 2012) to $174 \mathrm{mJy}$ Wright et al. (1994). Combining these values with the sensitivity limit of the USNO-B1 survey in this region ( $B \geq 20.8$ or $0.02 \mathrm{mJy}$ in flux), we estimate a radio loudness $\log \left(R_{L}\right) \geq 3$.7, which suggests that N2/PKS $1934-470$ is a radio loud AGN. The WISE magnitudes are $W_{1}=15.46, W_{2}=14.92, W_{3}=12.45$, and $W_{4}=8.85$ 
which constrains the WISE colours to be $W_{2}-W_{3}=2.45$ and $W_{1}-W_{2}=0.55$. This locates the source just outside the WBS.

In conclusion, we find that two sources can be responsible for the hard X-ray emission seen by INTEGRAL. Source N2 is very likely an AGN of still uncertain class, hence a valid counterpart, while less certain is the nature of source N1, which therefore remains a more dubious association.

\section{Conclusions}

We have used a well-tested cross-correlation technique to extract a sample of six unidentified/unclassified INTEGRAL objects that have a flat spectrum radio counterpart. This finding, together with the location above the galactic plane, suggests that they may all be AGN and therefore a valid association to the hard $\mathrm{X}$-ray emitters. To confirm these associations and to study the $\mathrm{X}$-ray properties of these objects, we made use of X-ray data collected by Swift/XRT through target of opportunity observations. Each source was analysed in detail using also archival multiwaveband data and information gathered from the literature.

We find that five of the six radio associations proposed in this work are also detected in X-rays; furthermore, in three cases they are the only counterpart found for the INTEGRAL source. More specifically, IGR J06073-0024 is a flat-spectrum radio quasar at high redshift, IGR J14488-4008 is a new radio galaxy with a complex morphology and a likely type 2 AGN nature, while IGR J18129-0649 is an AGN of still uncertain optical classification. The nature of IGR J07225-3810 and IGR J19386-4653 is defined less well, since we find in both cases another X-ray source in the INTEGRAL error circle; nevertheless the flat spectrum radio source, probably a radio-loud $A G N$, remains a viable and, in fact, more convincing association in both cases. Only for IGR J11544-7618 have we not been able to find a likely counterpart, since the radio association is not an X-ray emitter, while the only X-ray source seen in the field is a G star and therefore unlikely to produce persistent hard X-rays as observed by INTEGRAL.

Acknowledgements. This research has made use of data obtained from the SIMBAD database operated at the CDS, Strasbourg, France and from the High Energy Astrophysics Science Archive Research Center (HEASARC), provided by NASA's Goddard Space Flight Center NASA/IPAC Extragalactic Database (NED). The authors acknowledge financial support from the ASI under contracts ASI/033/10/0 and ASI/INAF I/009/10/0.

\section{References}

Arnaud, K. A. 1996, in Astronomical Data Analysis Software and Systems V, eds. G. H. Jacoby, \& J. Barnes, ASP Conf. Ser., 101, 17

Bennett, C. L., Lawrence, C. R., Burke, B. F., Hewitt, J. N., \& Mahoney, J. 1986, ApJS, 61, 1

Bird, A. J., Bazzano, A., Bassani, L., et al. 2010, ApJS, 186, 1

Bock, D. C.-J., Large, M. I., \& Sadler, E. M. 1999, AJ, 117, 1578

Burrows, D. N., Hill, J. E., Nousek, J. A., et al. 2005, Space Sci. Rev., 120, 165

Cohen, A. S., Lane, W. M., Cotton, W. D., et al. 2007, AJ, 134, 1245

Condon, J. J. 1992, ARA\&A, 30, 575

Condon, J. J., Cotton, W. D., Greisen, E. W., et al. 1998, AJ, 115, 1693

De Rosa, A., Panessa, F., Bassani, L., et al. 2012, MNRAS, 420, 2087

Douglas, J. N., Bash, F. N., Bozyan, F. A., Torrence, G. W., \& Wolfe, C. 1996, AJ, 111, 1945

Edelson, R., \& Malkan, M. 2012, ApJ, 751, 52

Gehrels, N., Chincarini, G., Giommi, P., et al. 2004, ApJ, 611, 1005

Ghisellini, G., Tavecchio, F., Foschini, L., et al. 2010, MNRAS, 402, 497

Griffith, M. R., Wright, A. E., Burke, B. F., \& Ekers, R. D. 1995, ApJS, 97, 347

Healey, S. E., Romani, R. W., Taylor, G. B., et al. 2007, ApJS, 171, 61

Hill, J. E., Burrows, D. N., Nousek, J. A., et al. 2004, in SPIE Conf. 5165, eds. K. A. Flanagan, \& O. H. W. Siegmund, 217

Kalberla, P. M. W., Burton, W. B., Hartmann, D., et al. 2005, A\&A, 440, 775

Kellermann, K. I., Sramek, R., Schmidt, M., Shaffer, D. B., \& Green, R. 1989, AJ, 98, 1195

Landi, R., Bassani, L., Malizia, A., et al. 2010, MNRAS, 403, 945

Landt, H., Padovani, P., Perlman, E. S., \& Giommi, P. 2004, MNRAS, 351, 83

Malizia, A., Landi, R., Bassani, L., et al. 2011, The Astronomer's Telegram, 3290, 1

Masetti, N., Parisi, P., Jiménez-Bailón, E., et al. 2012, A\&A, 538, A123

Massaro, E., Giommi, P., Leto, C., et al. 2009, VizieR Online Data Catalog: $\mathrm{J} / \mathrm{A}+\mathrm{A} / 495 / 691$

Massaro, F., D’Abrusco, R., Tosti, G., et al. 2012, ApJ, 752, 61

McConnell, D., Sadler, E. M., Murphy, T., \& Ekers, R. D. 2012, MNRAS, 422, 1527

Monet, D. G., Levine, S. E., Canzian, B., et al. 2003, AJ, 125, 984

Moretti, A., Campana, S., Tagliaferri, G., et al. 2004, in SPIE Conf. Ser. 5165, eds. K. A. Flanagan, \& O. H. W. Siegmund, 232

Murphy, T., Sadler, E. M., Ekers, R. D., et al. 2010, MNRAS, 402, 2403

Paturel, G., Petit, C., Prugniel, P., et al. 2003, A\&A, 412, 45

Skrutskie, M. F., Cutri, R. M., Stiening, R., et al. 2006, AJ, 131, 1163

Stephen, J. B., Bassani, L., Molina, M., et al. 2005, A\&A, 432, L49

Stephen, J. B., Bassani, L., Malizia, A., et al. 2006, A\&A, 445, 869

Stephen, J. B., Bassani, L., Landi, R., et al. 2010, MNRAS, 408, 422

Ubertini, P., Lebrun, F., Di Cocco, G., et al. 2003, A\&A, 411, L131

Vollmer, B. 2009, VizieR Online Data Catalog, 8085, 0

White, N. E., Giommi, P., \& Angelini, L. 2000, VizieR Online Data Catalog: IX/31

Winkler, C., Courvoisier, T. J.-L., Di Cocco, G., et al. 2003, A\&A, 411, L1

Wright, A. E., Griffith, M. R., Burke, B. F., \& Ekers, R. D. 1994, ApJS, 91, 111

Wright, E. L., Eisenhardt, P. R. M., Mainzer, A. K., et al. 2010, AJ, 140, 1868

Zombeck, M. V. 1990, Handbook of space astronomy and astrophysics, 2nd ed. (Cambridge: University Press) 